\title{
On Narrowband Interference Suppression in TDCS with WFRFT Preprocessing
}

\author{
Quan Wang $\mathbb{D}^{1}{ }^{1}$ Xinxi Feng, ${ }^{2}$ and Yuan Liang ${ }^{1}$ \\ ${ }^{1}$ Graduate School, Air Force Engineering University, Xi'an 710077, China \\ ${ }^{2}$ College of Information and Navigation, Air Force Engineering University, Xi'an 710077, China \\ Correspondence should be addressed to Quan Wang; wirture@gmail.com
}

Received 14 April 2018; Revised 16 July 2018; Accepted 19 July 2018; Published 15 August 2018

Academic Editor: Ruben Specogna

Copyright (c) 2018 Quan Wang et al. This is an open access article distributed under the Creative Commons Attribution License, which permits unrestricted use, distribution, and reproduction in any medium, provided the original work is properly cited.

\begin{abstract}
We propose a modified transform domain communication system (TDCS) structure with weighted fractional Fourier transform (WFRFT) preprocessing over the channel with additive white Gaussian noise (AWGN) and narrowband interference (NBI). The majority of NBI can be removed by adding zeros to TDCS's basic function (BF) amplitude vector at these subcarriers where strong NBI exists, and the corresponding residual NBI can be further greatly eliminated in time-frequency domain through WFRFT processing. To achieve the best bit error rate (BER) performance, we define $\gamma$ as the normalised cross relationship coefficient between TDCS's BF vector and residual NBI vector and prove that the corresponding WFRFT $\alpha_{\text {opt }}$ can be obtained by minimising the magnitude of $\gamma$. Numerical simulations are conducted to evaluate the proposed system's performance with regard to NBI suppression.
\end{abstract}

\section{Introduction}

In current carrier schemes, multicarrier (MC) and single carrier (SC) communication systems have become the two major techniques. To make best use of these two techniques' property, the long term evolution (LTE) system adopts orthogonal frequency division multiplexing (OFDM) for downlink transmission and single carrier frequency division multiple access (SC-FDMA) for uplink transmission [1]. Considering that OFDM and SC techniques have their individual advantages and disadvantages, researchers have done many attempts to converge these two carrier schemes [2-5]. Among them, the hybrid carrier (HC) system based on weighted-type fractional Fourier transform (WFRFT) is an inspiring attempt which can make a tradeoff between SC and MC. In addition, as a useful time-frequency analysing tool, WFRFT can make the original signal distributed more evenly in the time-frequency domain. The time-frequency distribution characteristics can greatly help mitigate intercarrier interference/intersymbol interference (ICI/ISI) [6] in additive white Gaussian noise (AWGN) or doubly selective channels [7] as long as the WFRFT order $\alpha$ is set properly.
Recently, our team has been extensively engaging in the related research on WFRFT. We explored WFRFT signals' inherent characteristics, such as modulation recognition [8] and antiparameter scanning ability $[9,10]$. Besides, we also demonstrated the feasibility and effectiveness of WFRFT's applications in physical layer (PHY) secure communications [11] and covert satellite communications [12-14]. In view of the above-mentioned facts, the WFRFT-based communication system will be a promising technology in the future communication system.

In the current broadband block-based transmission systems, most of them adopt the OFDM-based structure, then the WFRFT-based communication system also adopts the OFDM-based structure to be compatible with current communication structures. However, the OFDM-based communication systems are subject to the interference caused by narrowband interference (NBI), which has long attracted many researchers' attention $[15,16]$. As an important aspect of the anti-interference techniques, NBI suppression performance plays an important role in the system's performance evaluation. Thus, it is necessary to carry out research on NBI suppression in WFRFT-based systems. 
TABLE 1: Comparisons between different NBI suppression methods.

\begin{tabular}{|c|c|c|c|c|c|}
\hline Methods & Classification & Prior info. $(\mathrm{Y} / \mathrm{N})$ & $\begin{array}{c}\text { Iterative procedure } \\
(\mathrm{Y} / \mathrm{N})\end{array}$ & Suppression effects & $\begin{array}{c}\text { Mains ideas and } \\
\text { representative Refs }\end{array}$ \\
\hline $\begin{array}{l}\text { Successive } \\
\text { interference } \\
\text { cancellation }\end{array}$ & Subtraction & $\mathrm{N}$ & $\mathrm{Y}$ & A & $\begin{array}{c}\text { Successive } \\
\text { cancellation [19] / } \\
\text { Cancellation with soft } \\
\text { decision [20] }\end{array}$ \\
\hline Compressive Sense & Subtraction & Almost Y & $\mathrm{Y}$ & $\mathrm{A}$ & $\begin{array}{c}\text { A structured } \\
\text { dictionary Mismatch } \\
\text { formulation } \\
{[21] / \text { Sparse }} \\
\text { high-dimensional } \\
\text { NBI reconstruction } \\
{[22]} \\
\end{array}$ \\
\hline $\begin{array}{l}\text { Sparsity-Aware } \\
\text { approach/mitigation } \\
\text { based on CS }\end{array}$ & Subtraction & $\mathrm{N}$ & $\mathrm{Y}$ & A & $\begin{array}{c}\text { Inherent sparsity of } \\
\text { NBI [23]/Mitigating } \\
\text { spectral leakage to the } \\
\text { best [24] }\end{array}$ \\
\hline $\begin{array}{l}\text { Block sparse Bayesian } \\
\text { learning }\end{array}$ & Subtraction & $\mathrm{N}$ & $\mathrm{Y}$ & A & $\begin{array}{c}\text { Intra-block } \\
\text { correlation (IBC) [25] }\end{array}$ \\
\hline Equalization & Avoidance & $\mathrm{N}$ & $\mathrm{N}$ & B & $\begin{array}{c}\text { Linear precoding } \\
\text { [26]Maximum SNR } \\
\text { optimization } \\
\text { [27]/Widely } \\
\text { linear-zero force } \\
\text { [28]/Optimal rank } \\
\text { reduction [29] } \\
\end{array}$ \\
\hline Time/frequency filter & Avoidance & $\mathrm{N}$ & $\mathrm{N}$ & $\mathrm{B}$ & Excision filtering [30] \\
\hline Spread spectrum & Transform process & $\mathrm{N}$ & $\mathrm{N}$ & $\mathrm{C}$ & $\begin{array}{c}\text { Carrier } \\
\text { interferometry } \\
\text { spreading codes [31] }\end{array}$ \\
\hline Wavelet process & Transform process & $\mathrm{N}$ & $\mathrm{N}$ & $\mathrm{C}$ & $\begin{array}{c}\text { Wavelet domain } \\
\text { spectral periodogram } \\
{[32]}\end{array}$ \\
\hline $\begin{array}{l}\text { Frequency domain } \\
\text { process }\end{array}$ & Transform process & $\mathrm{N}$ & $\mathrm{N}$ & $\mathrm{C}$ & $\begin{array}{c}\text { Spectral shaping } \\
\text { [33]/Autoregressive } \\
\text { estimator to get PSD } \\
{[34]}\end{array}$ \\
\hline
\end{tabular}

In [17], it has been pointed out that WFRFT precoding structure can make the residual NBI distributed more evenly in the time-frequency domain and then eliminate the residual NBI to most extent. Furthermore, in [18], it has also been testified that WFRFT processing can help suppress NBI in the traditional direct sequence spread system (DSSS), while how to obtain the optimal BER performance on $\alpha$ has not been analysed.

To the best of our knowledge, according to the different ways to suppress the interference, these suppression methods can be classified into three categories: subtraction [19-25], avoidance [26-30], and transform process [31-34]. In order to have a more direct understanding of the differences between all kinds of NBI suppression methods in OFDM-based systems, we list a table to describe the comparisons between different NBI suppression methods, which are shown in Table 1. In Table 1, as for the suppression effects, to show how the specific methods can help eliminate the NBI, we define three levels, namely, "A", "B", and "C". "A", "B", and "C" denote the suppression effects "almost all", "a large amount", and "moderate", respectively.

The basic principle of subtraction methods is to detect the NBI's characteristic parameters and reconstruct the NBI based on these parameters. In particular, as for the compressive sense (CS) based subtraction methods, theoretically, NBI can be eliminated totally. However, as shown in Table 1, due to the reconstruction process, the prior information and iterative processes are often needed, which inevitably bring a great amount of computational burden. As for the avoidance methods, usually, by designing high performance filters to filter the NBI in time or frequency domain, the time/frequency domain filtering method can enhance the anti-interference property. In the meantime, it will also incur the performance reduction due to the useful signal's energy loss in the filter's stop bands and the high performance filters are also difficult to realise. Last, the transform process often does well in anti-NBI performance because of its capability to spread the interference into the corresponding transform 


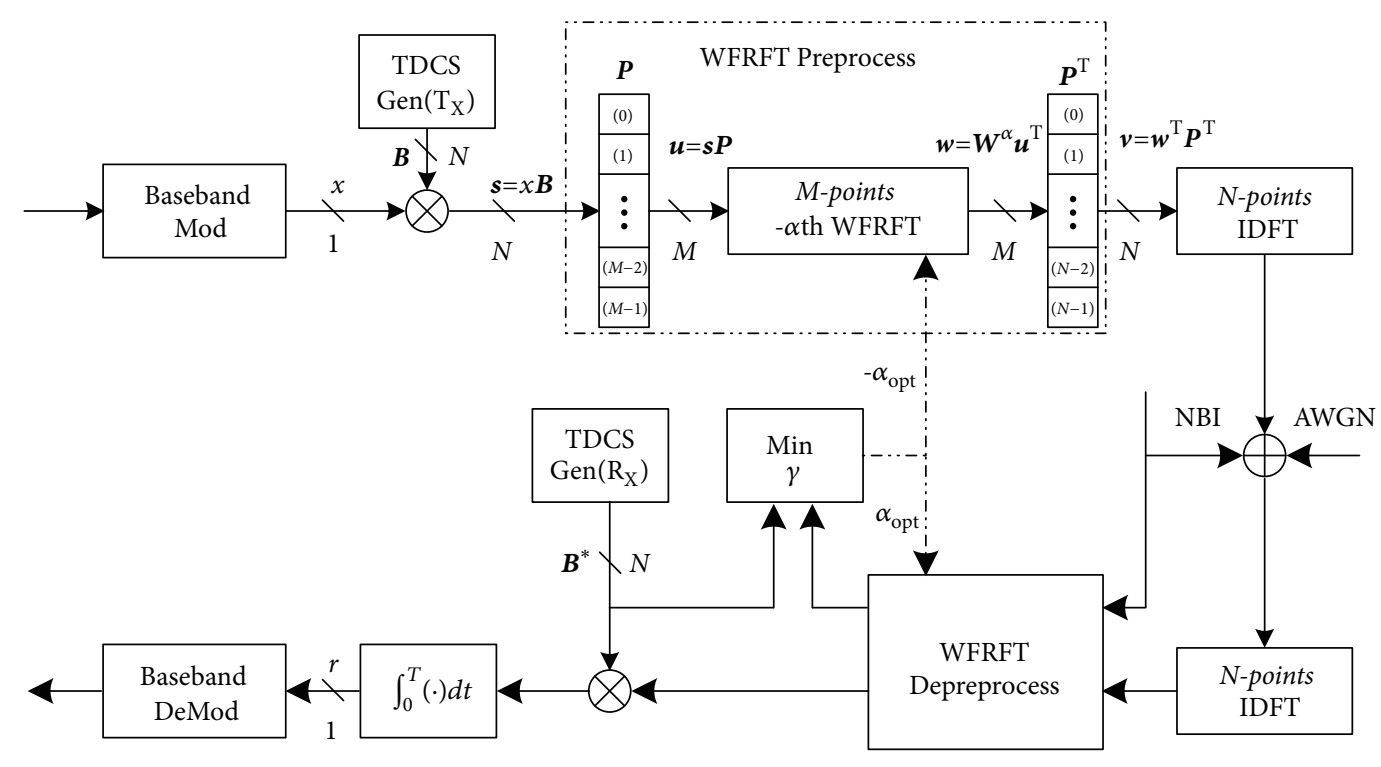

FIGURE 1: Overview structure of TDCS with WFRFT preprocessing.

domain. However, the universality of the transform process will also cause the insufficiency of NBI suppression.

Based on the above analyses, the traditional spread spectrum (SS) system has been proved an effective way to reduce the NBI or other interferences' effects on the system's bit error rate (BER) performance and has been widely used in the anti-interference communication system $[35,36]$. Moreover, by utilising the basic function (BF) to spread the original transmitting signal in a wider bandwidth [37, 38], transform domain communication system (TDCS) can serve as a special spread spectrum system in some sense [39, 40]. Although TDCS can avoid the majority of the NBI by zeroing the amplitude on these carriers position where strong interference exists, we cannot guarantee the NBI have been fully mitigated, given that the residual NBI is still distributed in the time-frequency domain unevenly.

Based on the theory mentioned above, in this paper, the main idea of the proposed structure is to combine the two methods, namely, avoidance and transform process. Compared with the subtraction method, the proposed structure can suppress the NBI to a great extent with a proper WFRFT order $\alpha$, while it will not cause too much implementation complexity. A modified TDCS structure with the WFRFT preprocessing technique is provided to make full use of the techniques of TDCS and WFRFT. The proposed system is motivated twofold. First, by adopting the traditional TDCS technique, we can remove the most part of NBI. Second, through WFRFT, we can make the residual NBI dispersed in a wider time-frequency domain, which helps mitigate the residual NBI to the greatest extent.

This paper will be organised as follows: In Section 2, the overview of the modified TDCS structure with WFRFT preprocessing is described in detail. In Section 3, through theoretical deductions, the system's BER performance has been derived, and the related principle has been established about how to select the optimal WFRFT order $\alpha_{\text {opt }}$. In Section 4, numerical simulations are conducted to evaluate the performance of the proposed system. Conclusions are drawn in the last section.

Notation. $\operatorname{Re}(\cdot), \operatorname{Im}(\cdot),(\cdot)^{*},(\cdot)^{\mathrm{T}}$, and $|\cdot|$ denote the real, imaginary, complex conjugate, transpose, and magnitude operations respectively. $\nabla P$ and $\nabla^{2} P$ are used to calculate the gradient and Hessian matrix of $P . \mathbf{I}, \mathbf{F}$, and $\mathbf{F}^{-1}$ represent the identity matrix, normalised discrete Fourier transform (DFT), and inverse discrete Fourier transform (IDFT), respectively. $u \sim U(a, b)$ means $u$ obeys the uniform distribution in $[a, b]$, and $n \sim N\left(\mu, \sigma^{2}\right)$ means $n$ obeys the normal distribution with mean $\mu$ and variance $\sigma^{2}$.

\section{System's Description}

Figure 1 illustrates the architecture of the proposed system combining TDCS spreading with WFRFT preprocessing. For description's clarity, we will use $\mathrm{Tx}$ and $\mathrm{Rx}$ to denote the transmitting and receiving parts, respectively.

As for $\mathrm{Tx}$, first, in the frequency domain expression, the basic function $(B F)$ vector $\mathbf{B}\left(\mathbf{B}=\left[A_{0} \mathrm{e}^{j 2 \pi \theta_{0}}, A_{1} \mathrm{e}^{j 2 \pi \theta_{1}}\right.\right.$, $\left.\left.\cdots, A_{N-1} \mathrm{e}^{j 2 \pi \theta_{N-1}}\right]\right)$ in TDCS is composed of the spectrum sense ID $\mathbf{A}\left(\mathbf{A}=\left[A_{0}, A_{1}, \cdots, A_{N-1}\right]\right)$ and pseudo phase vector $\boldsymbol{\theta}\left(\boldsymbol{\theta}=\left[\theta_{0}, \theta_{1}, \cdots, \theta_{N-1}\right]\right)$. Multiplied by the basic function vector $\mathbf{B}$, the original baseband signal $\mathbf{x}=\left[x_{0}\right.$, $\left.x_{1}, \cdots, x_{L-1}\right]$ can be transformed into $\mathbf{S}$, and $\mathbf{S}=\mathbf{x}^{\mathrm{T}} \mathbf{B}=$ $\left[x_{0} \mathbf{B}, x_{1} \mathbf{B}, \cdots, x_{L-1} \mathbf{B}\right]^{\mathrm{T}}$. As a spread spectrum technique, TDCS makes every element $x_{i}$ in $\mathbf{x}$ spread by the same BF vector $\mathbf{B}$, then we can omit the subscript $i$ for clarity's sake and pay close attention to the spread vector $\mathbf{s}=x \mathbf{B}$, where $x$ is a variable generated from $\mathbf{x}$.

Then through the frequency mapping and WFRFT preprocessing, the original signal can be transmitted through 


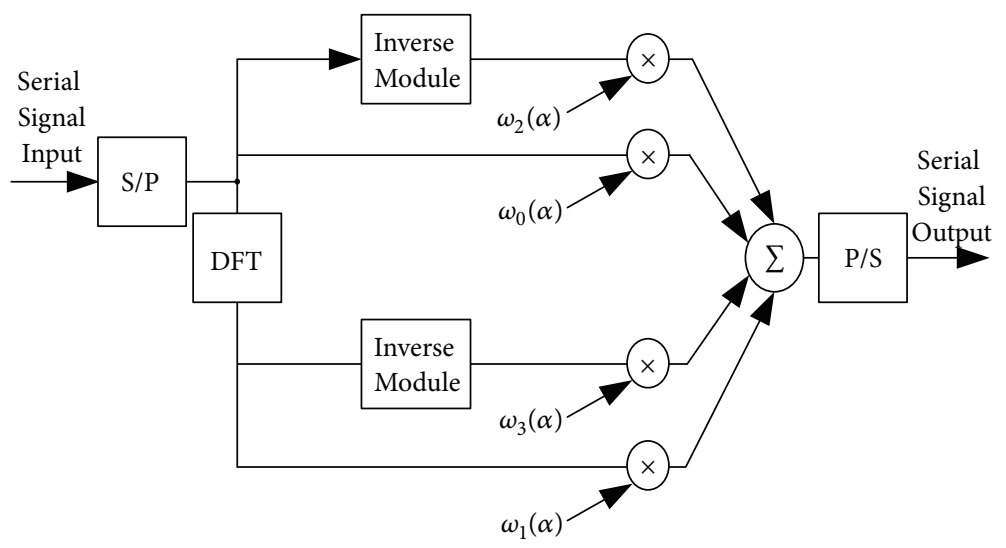

FIgURE 2: The implementation of WFRFT in DFT-based blocks.

the OFDM-based structure into the channel. As for $\mathrm{Rx}$, we assume the corresponding spectrum sense ID and the pseudo phase vector are the same with the Tx; besides, considering that the system has the corresponding inverse processing modules, the related descriptions about $\mathrm{Rx}$ are not presented in detail. The main work of this paper is to construct the related frequency mapping matrix and analyse how different WFRFT order can influence the designed system's capacity on NBI suppression. The channel is assumed to be only influenced by AWGN and NBI, so other adding cyclic prefix (CP) and frequency domain equalisation (FDE) modules are omitted. Detailed operating mechanism analyses on the designed system will be put as the following three subsections.

2.1. Spread Spectrum with TDCS. As for the BF vector B in Figure 1, it consists of the spectrum sense ID $\mathbf{A}$ and complex pseudo phase vector $\boldsymbol{\theta}$. By adding zeros to the amplitude vector $\mathbf{A}$ at these carriers where strong NBI exists, we can remove most of the NBI in its frequency domain. Besides, through a phase mapping from a pseudo-number (PN) sequence, such as $m$-sequence [41], $\boldsymbol{\theta}$ can be regarded as random variables obeying uniform distribution in $[0,2 \pi]$, $\theta_{i} \sim[0,2 \pi]$. Then the corresponding time-domain BF vector b can be given by

$$
\mathbf{b}=\mathbf{F}^{-1} \mathbf{B}=\mathbf{F}^{-1}(\mathbf{A} \odot \boldsymbol{\theta})
$$

where $\odot$ denotes the step-wise product.

According to the central limit theorem $[42,43]$, in a sense, b in (1) can be regarded as a Gaussian-like sequence and then can serve as a spreading sequence, just like the PN sequence in the spread spectrum (SS) system [38]. However, unlike the traditional SS, the majority of interference will first be suppressed and further be spread in the frequency domain.

2.2. Time-Frequency Domain Dispersion with WFRFT. In the matrix form, the normalised 4-WFRFT can be described as

$$
\mathbf{W}^{\alpha}=\omega_{0}(\alpha) \mathbf{F}^{0}+\omega_{1}(\alpha) \mathbf{F}^{1}+\omega_{2}(\alpha) \mathbf{F}^{2}+\omega_{3}(\alpha) \mathbf{F}^{3}
$$

where $\mathbf{F}$ is the $M$-point normalised DFT matrix, whose $(m, n)$-th element is $F_{m, n}=(1 / \sqrt{M}) \exp (-j * 2 \pi m n / M)$, $m, n=0,1, \cdots, M-1 ; \omega_{l}(\alpha)$ are weighting coefficients, and $\omega_{l}(\alpha)=(1 / 4) \sum_{n=0}^{3} \exp (j \pi n(\alpha-l) / 2), l=0,1,2,3$.

According to [17], the matrix $\mathbf{F}^{2}$ can be implemented through the shift matrix $\mathbf{T}$, whose expression is given by

$$
\mathbf{T}=\left[\begin{array}{cccccc}
1 & 0 & \cdots & 0 & 0 & 0 \\
0 & 0 & \cdots & 0 & 0 & 1 \\
0 & 0 & \cdots & 0 & 1 & 0 \\
\vdots & \cdots & 0 & \ddots & 0 & 0 \\
0 & 0 & 1 & 0 & \cdots & 0 \\
0 & 1 & 0 & 0 & \cdots & 0
\end{array}\right]
$$

Then, the WFRFT module can be implemented as Figure 2. As depicted in Figure 2, the S/P and P/S modules denote the serial-to-parallel and parallel-to-serial operations. And the DFT module and inverse module correspond matrix $\mathbf{F}$ and $\mathbf{T}$, respectively. Furthermore, in Figure 1, through WFRFT, $\mathbf{s}$ can be transformed into $\mathbf{w}$

$$
\begin{aligned}
\mathbf{w} & =\mathbf{W}^{\alpha}(\mathbf{u})^{\mathrm{T}}=\mathbf{W}^{\alpha}(\mathbf{s P})^{\mathrm{T}} \\
& =\left[\omega_{0}(\alpha) \mathbf{I}+\omega_{1}(\alpha) \mathbf{F}+\omega_{2}(\alpha) \mathbf{T}+\omega_{3}(\alpha) \mathbf{T F}\right](\mathbf{s} \mathbf{P})^{\mathrm{T}}
\end{aligned}
$$

where $\mathbf{P}$ is the frequency mapping matrix in Figure 1, details about $\mathbf{P}$ will be shown in next subsection. In $(4),\left[\omega_{1}(\alpha) \mathbf{F}+\right.$ $\left.\omega_{3}(\alpha) \mathbf{T F}\right](\mathbf{s P})^{\mathrm{T}}$ are the frequency domain parts and can be regarded as the multicarrier components; $\left[\omega_{0}(\alpha) \mathbf{I}+\right.$ $\left.\omega_{2}(\alpha) \mathbf{T}\right](\mathbf{s P})^{\mathrm{T}}$ are the time-domain parts and can be regarded as the single carrier components. That is the reason why WFRFT can make the original signal distributed in the timefrequency domain. Besides, different $\alpha$ in (4) will produce different distribution characteristics.

2.3. WFRFT Preprocessing Matrix Q. As illustrated in Figure 1 , to combine the traditional TDCS with WFRFT, we add the WFRFT preprocessing module between the modules IDFT and $\mathrm{BF}$ generator. In addition, to implement the elimination of the major NBI in TDCS, we utilise the frequency mapping 
matrix $\mathbf{P}$ and its transpose matrix $\mathbf{P}^{\mathrm{T}}$ to zero those carriers where strong NBI exists, and $\mathbf{P}$ is expressed by

$$
P_{i, j}= \begin{cases}1, & s_{i} \longmapsto u_{j} \\ 0, & \text { others }\end{cases}
$$

In (5), when the amplitude of NBI at the carrier frequency $i$ is larger than the preset threshold $\lambda$, the corresponding amplitude $A_{i}$ will be set to zero, and $P_{i, j}=0$; when the amplitude of NBI at the carrier frequency $i$ is less than the preset threshold $\lambda$, the corresponding amplitude $A_{i}$ will be set to one, and $P_{i, j}=1$, which means the $i$-th element in $\mathbf{s}$ will be mapped into the $j$-th element in $\mathbf{u}$.

As for Tx, the final transmitting signal $\mathbf{t}$ can be derived as

$$
\begin{aligned}
\mathbf{t} & =\mathbf{F}^{-1}(\mathbf{v})^{\mathrm{T}} \\
& =\mathbf{F}^{-1}(\mathbf{P} \mathbf{w}) \\
& =\mathbf{F}^{-1}\left(\mathbf{P} \mathbf{W}^{\alpha}(\mathbf{s} \mathbf{P})^{\mathrm{T}}\right) \\
& =\mathbf{F}^{-1}\left(\mathbf{P} \mathbf{W}^{\alpha} \mathbf{P}^{\mathrm{T}} \mathbf{s}^{\mathrm{T}}\right) \\
& =\mathbf{F}^{-1}\left(\mathbf{P} \mathbf{W}^{\alpha} \mathbf{P}^{\mathrm{T}}\right) \mathbf{B}^{\mathrm{T}} x
\end{aligned}
$$

To analyse the combinatorial effects caused by the related matrix $\mathbf{P}$ and $\mathbf{W}^{\alpha}$, for the sake of clarity, we defined the WFRFT preprocessing matrix $\mathbf{Q}$ as follows:

$$
\mathbf{Q}=\mathbf{P W}^{\alpha} \mathbf{P}^{\mathrm{T}}
$$

After substituting (7) into (6), we can rewrite (6) as

$$
\mathbf{t}=\mathbf{F}^{-1} \mathbf{Q B}^{\mathrm{T}} x
$$

As for $\mathrm{Rx}$, due to the corresponding inverse prepressing modules for TDCS and WFRFT, the original transmitting signal $x$ can be recovered to the most extent. However, when it comes to $\mathrm{NBI}$, the preprocessing matrix $\mathbf{Q}$ can eliminate the major part of NBI and further make the corresponding residual NBI dispersed in the time-frequency domain. Detailed analyses on the system's performance will be conducted in the next section.

\section{Theoretical Performance}

3.1. Anti-Gaussian Noise. As for Gaussian noise $\mathbf{n}(\mathbf{n}=$ $\left.\left[n_{0}, n_{1}, \cdots, n_{M-1}\right]^{\mathrm{T}}\right)$ and $n_{k} \sim N\left(\mu, \sigma^{2}\right)$ considering that the normalised 4-WFRFT is a unitary linear transformation, then $\mathbf{W}^{\alpha} \mathbf{n}$ have the same distribution properties with $\mathbf{n}$ [17]. Consider that the demapping matrix $\mathbf{Q}^{\mathrm{T}}$ will first zero some carrier frequencies and then make the residual NBI distributed in time-frequency domain. According to [44], through demapping with $\mathbf{Q}^{\mathrm{T}}$, the corresponding spread gain is $M$, and then the final signal-to-noise rate (SNR) can be expressed by

$$
\mathrm{SNR}=\left(\frac{\log 2\left(M_{\mathrm{PSK}}\right)}{M}\right) \cdot \frac{E_{\mathrm{b}}}{N_{0}}
$$

where $M_{\text {PSK }}$ is the baseband PSK modulation order and $E_{\mathrm{b}} / N_{0}$ is the original SNR without the spreading process with TDCS BF sequence.

3.2. Anti-NBI. In complex baseband form, the expression of $\mathrm{NBI} \mathbf{i}=\left[i_{0}, i_{1}, \cdots, i_{N-1}\right]^{\mathrm{T}}$ can be described as [45]

$$
\begin{aligned}
& i_{n}=\sum_{k=0}^{K-1} g_{k} \exp \left[j\left(\frac{2 \pi f_{0}(n-k T)}{N}+\varphi\right)\right] \\
& n=0,1, \cdots, N-1
\end{aligned}
$$

where $T$ is the period of each data symbol, $K$ is bandwidth ratio of wideband system to NBI, $K=\lceil N / T\rceil,\lceil A\rceil$ means to round the elements of $A$ to the nearest integers greater than or equal to $A, f_{0}$ denotes the central frequency of NBI, and $g_{k}$ denotes the $k$-th data symbol.

As for $\mathrm{Rx}$, through demapping matrix $\mathbf{Q}^{\mathrm{T}}$, for a symbol period ( $N$ points) in the designed system, the residual NBI signal can be given by

$$
\mathbf{i}_{\text {res }}=\mathbf{Q}^{\mathrm{T}} \mathbf{i}
$$

Then we can define NBI to signal ratio (ISR) within per bit with TDCS spreading as follows:

$$
\operatorname{ISR}=\sum_{n=0}^{N-1} \frac{\left|i_{n}\right|^{2}}{\left(N E_{b}\right)}
$$

Then, to analyse the effects of the $\mathbf{Q}^{\mathrm{T}}$ matrix and TDCS spreader to the residual NBI, we also define the crosscorrelation coefficient $\gamma$ between TDCS spreading sequence $\mathbf{B}$ and the residual NBI $\mathbf{i}_{\text {res }}$ as follows:

$$
\gamma=\sum_{n=0}^{N-1} i_{\text {res }, n} \cdot \frac{\left(B_{n}\right)^{*}}{\sqrt{\sum_{n=0}^{N-1}\left|i_{\text {res }, n}\right|^{2} \sum_{n=0}^{N-1}\left|B_{n}\right|^{2}}}
$$

where $i_{\text {res }, n}$ and $B_{n}$ correspond the $n$-th element of $\mathbf{i}_{\text {res }}$ and B, respectively. According to [46], when the residual NBI cannot be treated as Gaussian random variables, as for BPSK modulated system over channels with AWGN and other interference, the system's BER can be approximately be expressed as

$$
P=\frac{1}{2}\left\{Q\left[\frac{\sqrt{E_{b}}+\sqrt{E_{i} / 2}}{\sqrt{N_{0} / 2}}\right]+Q\left[\frac{\sqrt{E_{b}}-\sqrt{E_{i} / 2}}{\sqrt{N_{0} / 2}}\right]\right\}
$$

where $E_{b}, N_{0}$, and $E_{i}$ denote original signal energy and the noise power spectral density and the equivalent interference energy for one bit duration ( $N$ points), respectively, and $Q(x)$ is the complementary error function, whose expression is $Q(x)=(1 / \sqrt{2 \pi}) \int_{x}^{\infty} e^{-t^{2} / 2} \mathrm{~d} t$.

In the complex baseband forms, without loss of generality, we will take Gray-coded QPSK as the baseband modulation to deduce the related BER expression. As the real part and imaginary part of the Gray-coded QPSK baseband signal have the same BER performance with equal probability, the 
final BER conditioned on the residual NBI can be given by

$$
P=\frac{1}{4}\left\{\begin{array}{c}
Q\left[\sqrt{2 E_{b} / N_{0}}(1-\sqrt{\operatorname{ISR} R e}(\gamma))\right] \\
+Q\left[\sqrt{2 E_{b} / N_{0}}(1+\sqrt{\operatorname{ISR} R e}(\gamma))\right] \\
+Q\left[\sqrt{2 E_{b} / N_{0}}(1-\sqrt{\operatorname{ISR}} \operatorname{Im}(\gamma))\right] \\
+Q\left[\sqrt{2 E_{b} / N_{0}}(1+\sqrt{\operatorname{ISR} \operatorname{Im}}(\gamma))\right]
\end{array}\right\}
$$

where $E_{\mathrm{b}} / N_{0}$, ISR, and $\gamma$ are corresponding to (9), (12), and (13), respectively.

3.3. Optimal Order Selection. As for the optimisation program in (15), when NBI, ISR, and SNR are determined, BER will just be the function of $\alpha$. Different WFRFT order $\alpha$ will produce different BER performances. Through strict mathematic deductions, we will prove that the optimal WFRFT order $\alpha_{\text {opt }}$ can be obtained by minimising the magnitude of $\gamma$ in (15). The related deduction is put as follows.

Let $x=|\operatorname{Re}(\gamma)|, y=|\operatorname{Im}(\gamma)|$, and $x, y \geq 0$. Using derivative method, $\nabla P=[\partial P / \partial x, \partial P / \partial y]^{T}=0$, then we can get the following expressions:

$$
\begin{aligned}
& \left\{\begin{array}{l}
\sqrt{\frac{2 E_{b}}{N_{0}}} \sqrt{\operatorname{ISR}}\left(\begin{array}{c}
e^{-0.5\left[\sqrt{2 E_{b} / N_{0}}(1-\sqrt{\operatorname{ISR}} x)\right]^{2}} \\
-e^{-0.5\left[\sqrt{2 E_{b} / N_{0}}(1+\sqrt{\operatorname{ISR}} x)\right]^{2}}
\end{array}\right)=0 \\
\sqrt{\frac{2 E_{b}}{N_{0}}} \sqrt{\operatorname{ISR}}\left(\begin{array}{c}
e^{-0.5\left[\sqrt{2 E_{b} / N_{0}}(1-\sqrt{\operatorname{ISR} y})\right]^{2}} \\
-e^{-0.5\left[\sqrt{2 E_{b} / N_{0}}(1+\sqrt{\operatorname{ISR}} y)\right]^{2}}
\end{array}\right)=0
\end{array}\right. \\
& \Longrightarrow\left\{\begin{array}{l}
\sqrt{\operatorname{ISR}} x=0 \\
\sqrt{\operatorname{ISR}} y=0
\end{array}\right.
\end{aligned}
$$

Usually, ISR is a positive number, then the equation $|\gamma|=0$ is equivalent to the one $x=0, y=0$. When $|\gamma| \neq 0$, that is to say, $x, y>0$, according to (16), we can easily find that $\partial P / \partial x>0$, $\partial P / \partial y>0$. Then according to [47], we can achieve the local minimum of $P$ in (15) when $|\gamma|=0(x=0, y=0)$.

Meanwhile, we can calculate the Hessian matrix for $P$ in (15) as follows:

$$
\begin{aligned}
& \nabla^{2} P=\left[\begin{array}{l}
\frac{\partial^{2} P}{\partial x^{2}}, \frac{\partial^{2} P}{\partial y \partial x} \\
\frac{\partial^{2} P}{\partial x \partial y}, \frac{\partial^{2} P}{\partial y^{2}}
\end{array}\right] \\
& =\left[\begin{array}{c}
\frac{2 E_{b}}{N_{0}} \cdot \operatorname{ISR} \cdot\left[\begin{array}{c}
(1-\sqrt{\operatorname{ISR} y}) e^{-0.5\left[\sqrt{2 E_{b} / N_{0}}(1-\sqrt{\operatorname{ISR}} y)\right]^{2}} \\
\left.+(1+\sqrt{\operatorname{ISR} y}) e^{-0.5\left[\sqrt{2 E_{b} / N_{0}}(1+\sqrt{\operatorname{ISR}} y)\right]^{2}}\right)
\end{array}\right], 0 \\
0, \frac{2 E_{b}}{N_{0}} \cdot \operatorname{ISR} \cdot\left[\begin{array}{c}
(1-\sqrt{\operatorname{ISR} y}) e^{-0.5\left[\sqrt{2 E_{b} / N_{0}}(1-\sqrt{\operatorname{ISR}} y)\right]^{2}} \\
\left.+(1+\sqrt{\operatorname{ISR}} y) e^{-0.5\left[\sqrt{2 E_{b} / N_{0}}(1+\sqrt{\operatorname{ISR}} y)\right]^{2}}\right)
\end{array}\right]
\end{array}\right]
\end{aligned}
$$

After substituting $x=0, y=0$ into (17), we can get the following expression:

$$
\nabla^{2} P=\left[\begin{array}{l}
\frac{2 E_{b}}{N_{0}} \cdot \operatorname{ISR} \cdot 2 e^{-0.5^{2}}, 0 \\
0, \frac{2 E_{b}}{N_{0}} \cdot \operatorname{ISR} \cdot 2 e^{-0.5^{2}}
\end{array}\right]
$$

Obviously, the Hessian matrix $\nabla^{2} P$ in (18) is positive definite according to the convex optimisation theory [48]; the local minimum at $|\gamma|=0(x=0, y=0)$ is the global minimum in (15). Meanwhile, $|\gamma|=0$ means that the residual NBI signal $\mathbf{i}_{\text {res }}$ and the BF vector $\mathbf{B}$ are totally unrelated to each other, which is almost impossible to make it in the actual communications. However, based on the analysis of (15)-(18), we can still draw a conclusion that there exists an obvious positive correlation between the designed system's BER $P$ and the cross-correlation coefficient magnitude $|\gamma|$. So, to get the best resistance to the residual NBI in the designed system, we can keep $|\gamma|$ as small as possible by selecting the WFRFT order $\alpha$ properly.

Based on the theoretical deduction mentioned above, the optimal program of (15) can simply come down to the following optimisation:

$$
\alpha_{\mathrm{opt}}=\arg \min _{\alpha \in[0,1]}\left(x^{2}+y^{2}\right)=\arg \min _{\alpha \in[0,1]}|\gamma|
$$

As shown in (19), to be compatible with the current SC and MC system, we only search the optimal $\alpha$ in the range $[0,1]$ even though the classical WFRFT order have a period of 4 . When $\alpha=0$, the TDCS with WFRFT preprocessing can degrade into the traditional TDCS system with the OFDMbased structure in [49], and when $\alpha=1$, the designed system operates with a DFT-preprocessing, similar to the mechanism in the SC-FDMA system. Certainly, we can utilise the exhaustive searching method or other fast iterative searching methods to get the optimal WFRFT order $\alpha_{\text {opt }}$ from 0 to 1.

\section{Simulations and Discussion}

To test the performance of the designed system in Figure 1 and validate the related theoretical analyses in Section 3, we conduct the following simulations with TDCS block length $N=512$ and Gray-coded QPSK baseband modulation. We assume that the channel is only influenced by AWGN and NBI. As for basic function (BF) in TDCS, the pseudo random phase is generated by a phase mapper from a $m$-sequence generator. And as for NBI in (10), the spreading factor $K$ is set to 32 , and the central frequency $f_{0}$ is set to 0.3 normalised block length. $g_{k}$ obeys Bernoulli distribution, and the initial phase $\varphi$ is uniformly distributed in $[0,2 \pi]$. The threshold for TDCS $\lambda$ is set to $0.4 * J_{\max }$, and $J_{\max }$ is the maximum spectrum amplitude for NBI.

4.1. Time-Frequency Distribution. To analyse how WFRFT can influence the signal's distribution in time-frequency domain, we illustrate different residual NBI's amplitude distribution with different $\alpha$. As shown in Figure 3, for these samples in frequency domain where the corresponding amplitude is larger than the threshold $\lambda$ (ZERO interval in each subfigures), the WFRFT preprocessing matrix $\mathbf{Q}$ in (7) can always zero the amplitudes at these carrier frequencies to remove the most strong interference of NBI. As depicted in Figures 3(a)-3(d), different $\alpha$ in $\mathbf{Q}$ will make the different residual NBI's distribution in the other nonzero carriers 


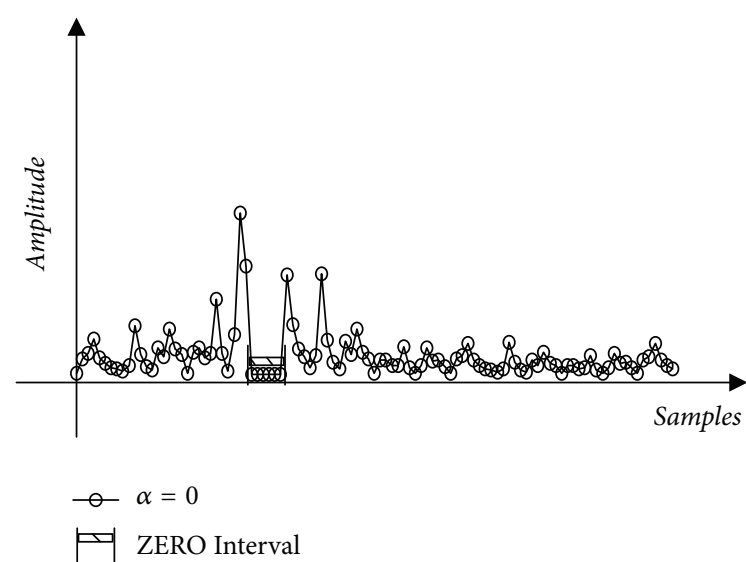

(a)

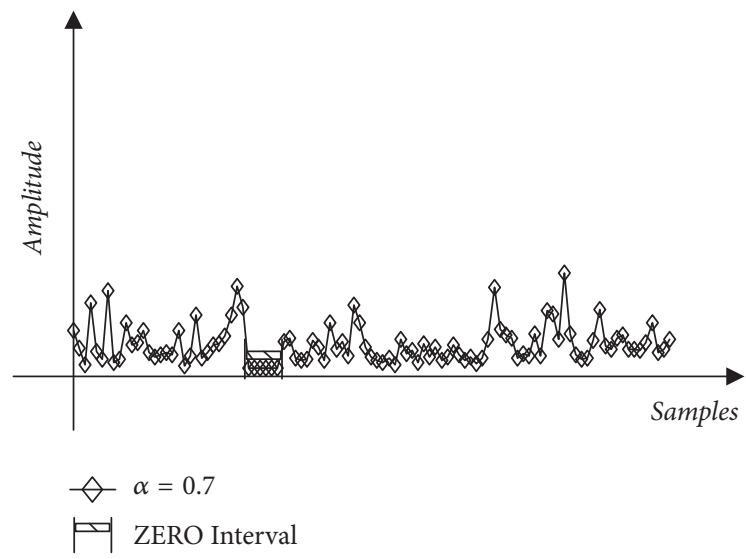

(c)

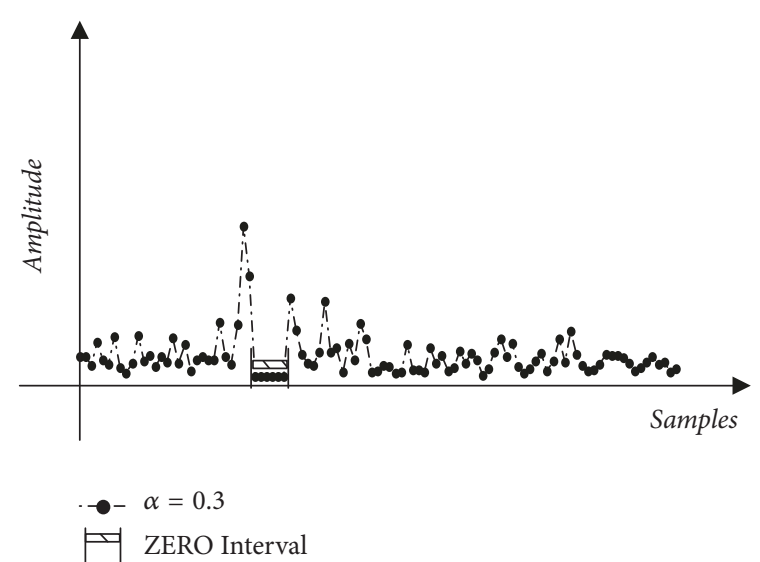

(b)

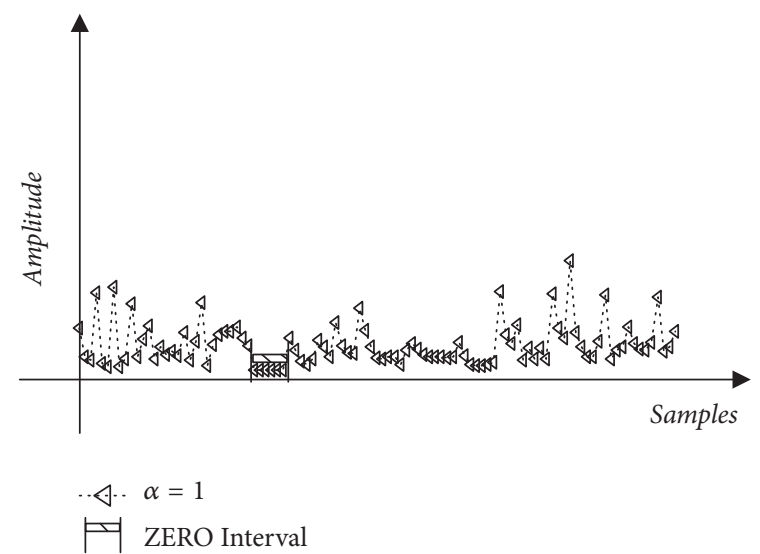

(d)

FIGURE 3: Residual NBI's amplitude distributions with different WFRFT order $\alpha$.

position, which can further suppress the residual NBI to some extent.

\subsection{BER Performance with JSR and SNR. To test the designed} system's BER performance of different $\alpha$ selections against SNR and ISR, we set SNR in the range $[1,8] \mathrm{dB}$ and ISR in the range $[8,25] \mathrm{dB}$. And the BER performances are averaged over 1000 Monte Carlo trails. Besides, exhausted searching method is adopted to get the optimal $\alpha_{\text {opt }}$ in (19), and optimal BER performance can be achieved at $\alpha=$ 0.3 for the current simulation conditions. In Figure 4 , the BER performance curves with three typical $\alpha$ selections are exhibited to compare the suppression performances on the NBI. As depicted in Figure 4 , the system at $\alpha=0.3$ can greatly outperform the ones at both $\alpha=0$ and $\alpha=1$ with regard to NBI suppression. When ISR is larger enough, for example, when ISR $>25 \mathrm{~dB}$, the system with $\alpha=0.3$ can gain $3-10 \mathrm{~dB}$ than the one at $\alpha=0$ and $1\left(\mathrm{SNR}=8 \mathrm{~dB}\right.$, and $\left.\mathrm{BER}=4.5 * 10^{-4}\right)$. This is mainly because $\sqrt{\text { ISR }} *|\gamma|$ can be regarded as the major factor to affect the BER performance in (15), while $|\gamma|$ can be reduced to the most extent at $\alpha=0.3$. When ISR $<$ $14 \mathrm{~dB}$, there exists small difference between the systems with different $\alpha$ selections, and the performance difference can be neglected especially when SNR is small enough ( SNR $<8 \mathrm{~dB})$. This is mainly because the Gaussian noise has been the major factor to affect the final BER performance, and the NBI's factor $\sqrt{\mathrm{ISR}} *|\gamma|$ can be omitted compared to the Gaussian noise factor $\sqrt{2 E_{b} / N_{0}}$ in (15).

4.3. Cross Relationship Coefficient. We also test the normalised cross-correlation coefficients $\gamma$ in (13) between residual NBI ( $\left.\mathbf{i}_{\text {res }}\right)$ and BF (B) with different central frequency, $f_{0}$. As shown in Figure 5 , different central frequency $f_{0}$ will result in different optimisation of $\gamma$. This mainly because the final vectors for BF (B) in (1) and residual NBI ( $\left.\mathbf{i}_{\text {res }}\right)$ in (11) will also change when the amplitude vector $\mathbf{A}$ change with different $f_{0}$.

Besides, different vector for residual NBI will further result in quite different time-frequency domain distributions of residual NBI, just shown in Figure 4. The simulation results in Figure 5 show that the optimal WFRFT order $\alpha_{\text {opt }}$ should be decided and varies with the different NBI's central frequency $f_{0}$. As shown in Figure 5 , especially when $f_{0}=$ 0.3 , the optimal WFRFT order $\alpha_{\text {opt }}$ is 0.3 , and the current optimal result is just consistent with the BER performances in Figure 4, which further demonstrates the correctness of the related optimal theory in (19). 


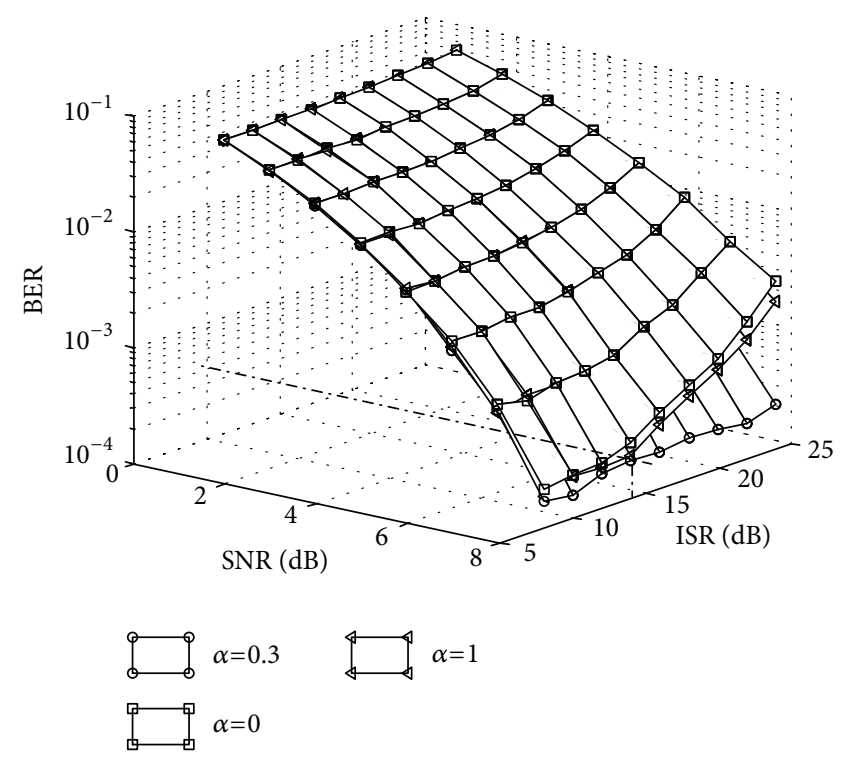

FIGURE 4: BER comparison of the proposed system.

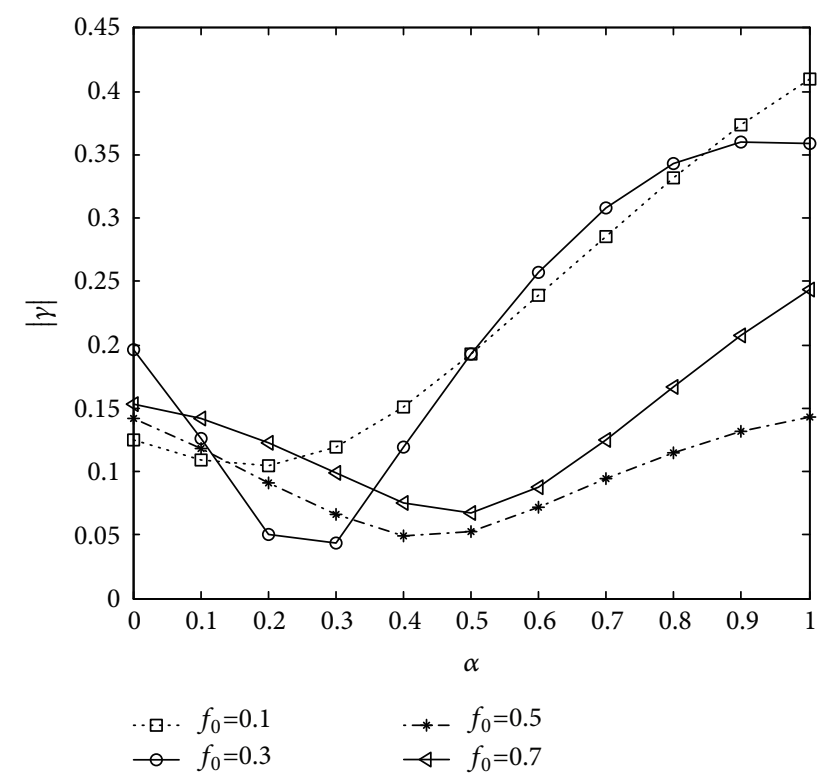

FIGURE 5: Optimal order selections for different central frequency $f_{0}$.

\section{Conclusions}

In this paper, we provide a modified TDCS structure with WFRFT preprocessing and analyse the system's ability on NBI suppression. Through theoretical deduction, we also derive the system's BER expression and prove that the best BER's performance could be obtained by minimising the magnitude of the cross relationship coefficient $\gamma$ between TDCS's basic function vector and residual NBI vector. Compared with the traditional TDCS structure, final simulation results demonstrate that the modified structure with the optimal WFRFT order can exhibit best BER performance to suppress NBI to most extent. As for the optimal program in (19), the exhausted searching method is adopted in the simulation.
Thus, in our future works, with regard to WFRFT order $\alpha$ selection, the highly efficient searching algorithms need to be explored to achieve the optimal BER performance more effectively. Besides, to further evaluate the proposed system's practicability, we will also proceed with analysis on the system's anti-interception ability and antimultipath performances.

\section{Data Availability}

The data sets supporting the results of the article are included within the article and its additional files.

\section{Conflicts of Interest}

The authors declare that there are no conflicts of interest regarding the publication of this paper.

\section{Acknowledgments}

This work was supported by the National Natural Science Foundation of China (Grant 61271250) and the National Natural Science Foundation of China (Grant 61571460).

\section{References}

[1] B. Furht and S. A. Ahson, Long Term Evolution: 3GPP LTE radio and cellular technology, Crc Press, 2nd edition, 2016.

[2] X.-G. Xia, "Precoded and vector OFDM robust to channel spectral nulls and with reduced cyclic prefix length in single transmit antenna systems," IEEE Transactions on Communications, vol. 49, no. 8, pp. 1363-1374, 2001.

[3] N. Michailow, M. Matthe, I. S. Gaspar et al., "Generalized frequency division multiplexing for 5 th generation cellular networks," IEEE Transactions on Communications, vol. 62, no. 9, pp. 3045-3061, 2014.

[4] I. Kashiwamura, S. Tomita, K. Komatsu et al., "Investigation on single-carrier and multi-carrier hybrid system for uplink," in Proceedings of the 2009 IEEE 20th Personal, Indoor and Mobile Radio Communications Symposium, PIMRC 2009, Japan, September 2009.

[5] L. Mei, X.-J. Sha, and N.-T. Zhang, "The approach to carrier scheme convergence based on 4-weighted fractional fourier transform," IEEE Communications Letters, vol. 14, no. 6, pp. 503-505, 2010.

[6] S. Yu, H. Dai, K. Wu, G. Zhou, X. Cheng, and C. Xu, "Performance analysis for WFRFT-OFDM systems to carrier frequency offset in doubly selective fading channels," in Proceedings of the 6th International Conference on Intelligent Control and Information Processing, ICICIP 2015, pp. 6-10, China, November 2015.

[7] Y. Hui, B. Li, and Z. Tong, "4-weighted fractional Fourier transform over doubly selective channels and optimal order selecting algorithm," IEEE Electronics Letters, vol. 51, no. 2, pp. 177-179, 2015.

[8] Y. Liang, X. Da, R. Xu, Z. Zhang, and H. Liu, "WFRFT modulation recognition based on HOC and optimal order searching algorithm," Journal of Systems Engineering and Electronics, vol. 29, no. 3, pp. 462-470, 2018. 
[9] Y. Liang, X. Da, R. Xu, and L. Ni, "Design of constellation precoding in MP-WFRFT based system for covert communications," Journal of Huazhong University of Science and Technology, vol. 46, no. 2, pp. 72-78, 2018.

[10] Y. Liang and X. Da, "Analysis and implementation of constellation precoding system based on multiple parameters weightedtype fractional Fourier transform," Journal of Electronics Information Technology, vol. 40, no. 4, pp. 825-831, 2018.

[11] L. Ni, X. Da, S. Wang, and Y. Liang, "Rsearch on satellite covert conmmunication based on the information encryption of physical layer," Advanced Engineering Sciences, vol. 50, no. 1, pp. 133-139, 2018.

[12] Z. Zhang, X. Da, H. Liu, and Y. Liang, "A secure transmission scheme for satellite communications based on hybrid carrier and chaotic phase scrambling," Journal of Xian Jiaotong University, vol. 51, no. 12, pp. 42-48, 2017.

[13] Z. Zhang, X. Da, and H. Liu, "A study of the covered characteristics of MAP-WFRFT satellite signals," Journal of Chongqing University of Posts Telecommunications, vol. 29, no. 4, pp. 460467, 2017.

[14] H. Liu, X. Da, and Z. Zhang, "Satellite covert communication system with DL-MPWFRFT and constellation scrambling," Journal of Air Force Engineering University (Natural Science Edition), vol. 18, no. 5, pp. 73-79, 2017.

[15] S. B. Weinstein, "The history of orthogonal frequency-division multiplexing," IEEE Communications Magazine, vol. 47, no. 11, pp. 26-35, 2009.

[16] M. Morelli and M. Moretti, "Improved decoding of BICMOFDM transmissions plagued by narrowband interference," IEEE Transactions on Wireless Communications, vol. 10, no. 1, pp. 20-26, 2011.

[17] L. Mei, Q. Zhang, X. Sha, and N. Zhang, "WFRFT precoding for narrowband interference suppression in DFT-based block transmission systems," IEEE Communications Letters, vol. 17, no. 10, pp. 1916-1919, 2013.

[18] X.-J. Sha, X. Qiu, and L. Mei, "Hybrid carrier CDMA communication system based on weighted-type fractional fourier transform," IEEE Communications Letters, vol. 16, no. 4, pp. 432-435, 2012.

[19] D. Darsena, "Successive narrowband interference cancellation for OFDM systems," IEEE Communications Letters, vol. 11, no. 1, pp. 73-75, 2007.

[20] D. Darsena and F. Verde, "Successive NBI cancellation using soft decisions for OFDM systems," IEEE Signal Processing Letters, vol. 15, pp. 873-876, 2008.

[21] H. Al-Tous, I. Barhumi, and N. Al-Dhahir, "Narrow-Band Interference Mitigation Using Compressive Sensing for AFOFDM Systems," IEEE Transactions on Vehicular Technology, vol. 66, no. 7, pp. 6146-6159, 2017.

[22] S. Liu, F. Yang, and J. Song, "Narrowband interference cancelation based on priori aided compressive sensing for DTMB systems," IEEE Transactions on Broadcasting, vol. 61, no. 1, pp. 66-74, 2015.

[23] A. Gomaa and N. Al-Dhahir, "A sparsity-aware approach for NBI estimation in MIMO-OFDM," IEEE Transactions on Wireless Communications, vol. 10, no. 6, pp. 1854-1862, 2011.

[24] K.-H. Kim, "NBI spectral leakage mitigation based on compressed sensing in OFDM systems," IEEE Transactions on Broadcasting, vol. 62, no. 2, pp. 409-416, 2016.
[25] S. Liu, F. Yang, J. Song, and Z. Han, "Block Sparse Bayesian Learning Based NB-IoT Interference Elimination in LTEAdvanced Systems," IEEE Transactions on Communications, vol. 65, no. 10, pp. 4559-4571, 2017.

[26] D. Darsena, G. Gelli, and F. Verde, "Universal linear precoding for NBI-proofwidely linear equalization in MC systems," EURASIP Journal on Wireless Communications and Networking, vol. 2008, no. 1, pp. 1-13, 2008.

[27] D. Darsena, G. Gelli, L. Paura, and F. Verde, "A constrained maximum-SINR NBI-resistant receiver for OFDM systems," IEEE Transactions on Signal Processing, vol. 55, no. 6, pp. 30323047, 2007.

[28] D. Darsena, G. Gelli, L. Paura, and F. Verde, "Widely linear equalization and blind channel identification for interferencecontaminated multicarrier systems," IEEE Transactions on Signal Processing, vol. 53, no. 3, pp. 1163-1177, 2005.

[29] R. Nilsson, F. Sjöberg, and J. P. LeBlanc, "A rank-reduced LMMSE canceller for narrowband interference suppression in OFDM-based system," IEEE Transactions on Communications, vol. 51, no. 12, pp. 2126-2140, 2003.

[30] A. J. Coulson, "Bit error rate performance of OFDM in narrowband interference with excision filtering," IEEE Transactions on Wireless Communications, vol. 5, no. 9, pp. 2484-2492, 2006.

[31] Z. Wu and C. R. Nassar, "Narrowband interference rejection in OFDM via carrier interferometry spreading codes," IEEE Transactions on Wireless Communications, vol. 4, no. 4, pp. 14911505, 2005.

[32] Y. Jo and D. Wu, "An enhanced transform domain communication system (ETDCS) with narrow-band interference (NBI) avoidance capability," in Proceedings of the Sensors, and Command, Control, Communications, and Intelligence (C3I) Technologies for Homeland Security and Homeland Defense VIII, USA, April 2009.

[33] J. Zhang and J. Meng, "Noise resistant OFDM for power-line communication systems," IEEE Transactions on Power Delivery, vol. 25, no. 2, pp. 693-701, 2010.

[34] K. Tan, J. Andrian, F. Candocia, and C. Zhou, "An Enhanced Wavelet Domain Communication System (EWDCS) with Nonstationary Interference Avoidance Capability," in Proceedings of the IEEE Vehicular Technology Conference, pp. 1-6, Hyatt Regency Montreal, Montreal, QC, Canada, September 2006.

[35] L.-L. Guo, F.-L. Yin, and M.-H. Lu, "Overview on NBI suppression of DSSS/CDMA systems," Tien Tzu Hsueh Pao/Acta Electronica Sinica, vol. 37, no. 10, pp. 2248-2257, 2009.

[36] X. Wu and Z. Yang, "Coding versus spreading for narrowband interference suppression," IEEE Transactions on Vehicular Technology, vol. 65, no. 4, pp. 2129-2141, 2016.

[37] E. H. German, "Transform domain signal processing study final report, Tech. rep," Air Force, pp. F30602-86, 1988.

[38] R. A. Radcliffe and C. G. Gerald, "Design and simulation of a transform domain communication system," in Proceedings of the MILCOM, p. 1, 1997.

[39] G. Fumat, P. Chargé, A. Zoubir, and D. Fournier-Prunaret, "Transform domain communication systems from a multidimensional perspective, impacts on bit error rate and spectrum efficiency," IET Communications, vol. 5, no. 4, pp. 476-483, 2011.

[40] C. Chang, H. Huan, J. Guo, and R. Tao, "Complementary peak reducing signals for TDCS PAPR reduction," IET Communications, vol. 11, no. 6, pp. 961-967, 2017.

[41] P. J. Swackhammer, M. A. Temple, and R. A. Raines, "Performance simulation of a transform domain communication 
system for multiple access applications," in Proceedings of the IEEE Military Communications Conference (MILCOM 1999), pp. 1055-1059, November 1999.

[42] W. Akmouche, "Detection of multicarrier modulations using 4th-order cumulants," in Proceedings of the IEEE Military Communications Conference (MILCOM 1999), pp. 432-436, November 1999.

[43] M. Pätzold, Mobile Fading Channels, John Wiley \& Sons, Ltd, Chichester, UK, 2002.

[44] C. Han, J. Wang, Y. Yang, and S. Li, "Addressing the control channel design problem: OFDM-based transform domain communication system in cognitive radio," Computer Networks, vol. 52, no. 4, pp. 795-815, 2008.

[45] M. E. Şahin, I. Guvenc, and H. Arslan, "An iterative interference cancellation method for co-channel multicarrier and narrowband systems," Physical Communication, vol. 4, no. 1, pp. 13-25, 2011.

[46] C. Zhang, H. Yin, and P. Ren, "The effects of narrowband interference on finite-resolution IR-UWB digital receivers," IEEE Communications Letters, vol. 15, no. 5, pp. 536-538, 2011.

[47] H. hima and T. Nakayama, Higher mathematics for physics and engineering, Springer Science Business Media, 1st edition, 2010.

[48] C. A. Floudas and P. M. Pardalos, Encyclopedia of Optimization, Springer US, Boston, MA, 2001.

[49] C. Han, J. Wang, S. Gong, and S. Li, "Performance of the OFDM-based transform domain communication system in cognitive radio contexts," in Proceedings of the 1st International Conference on Cognitive Radio Oriented Wireless Networks and Communications 2006, CROWNCOM, Greece, June 2006. 


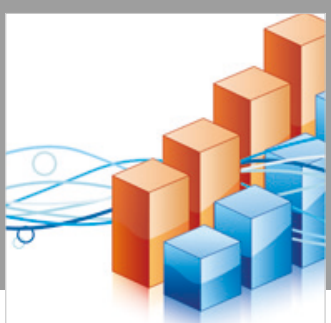

Advances in

Operations Research

\section{-n-m}
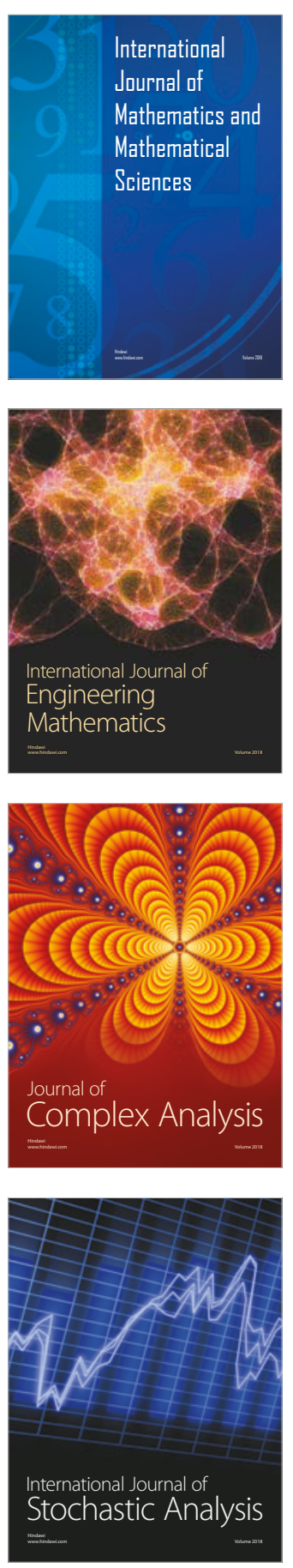
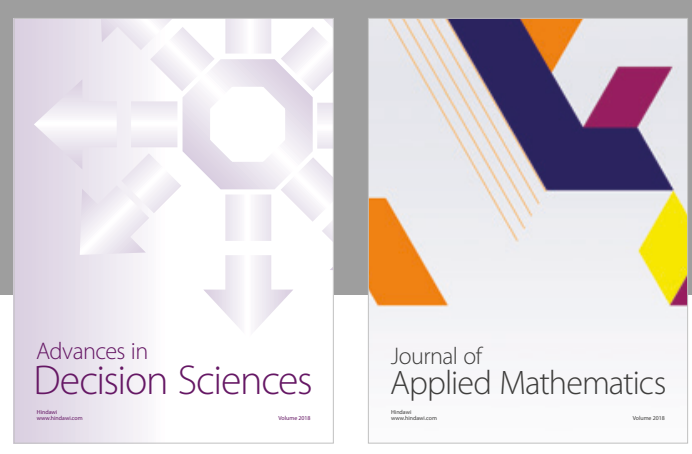

Journal of

Applied Mathematics
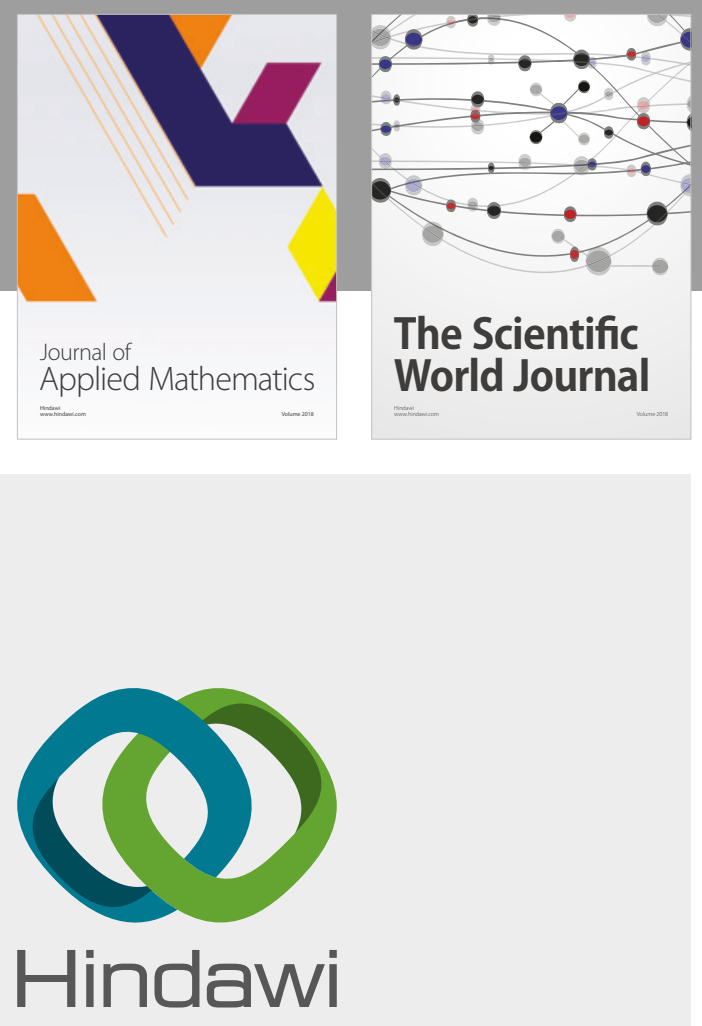

Submit your manuscripts at

www.hindawi.com

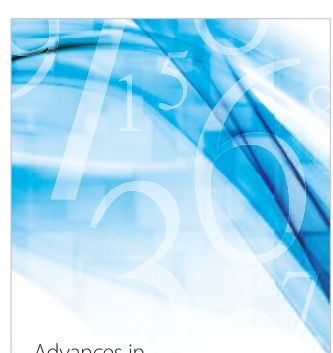

Advances in
Numerical Analysis
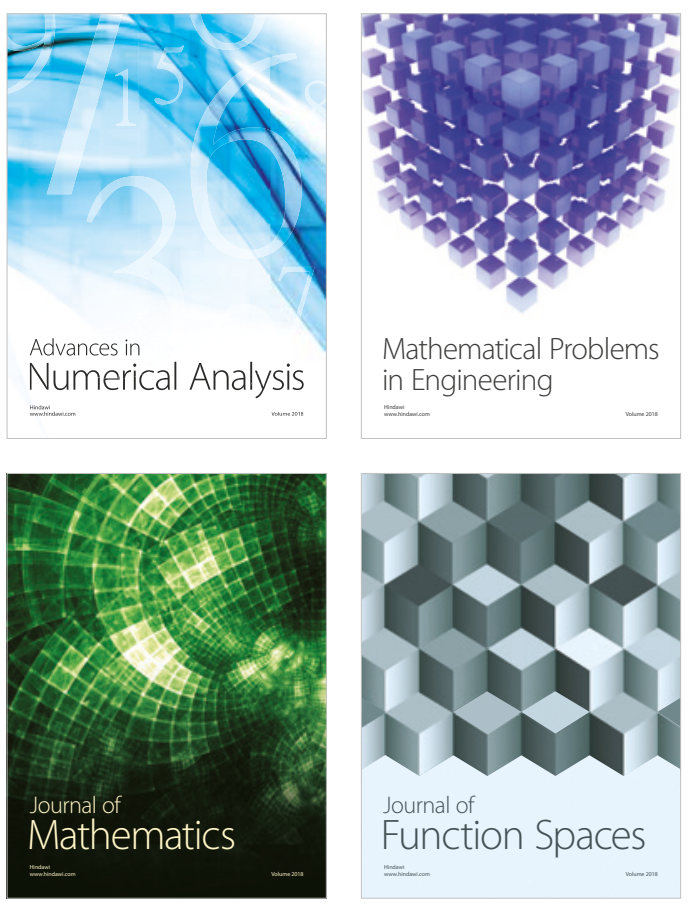

Mathematical Problems in Engineering

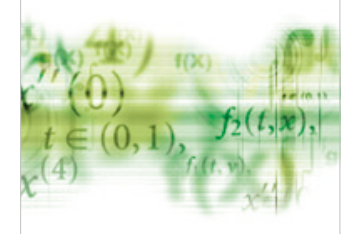

International Journal of

Differential Equations

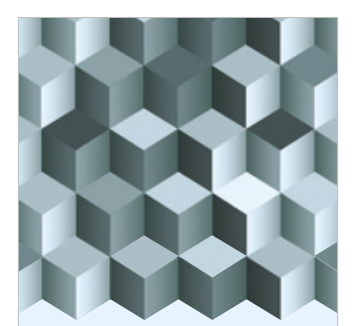

Journal of

Function Spaces

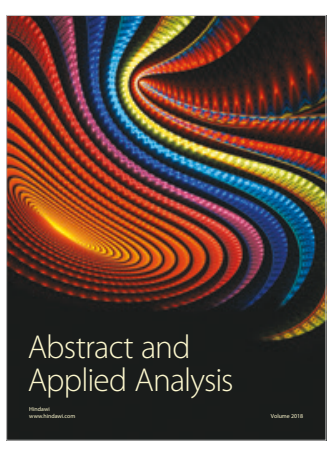

The Scientific

World Journal

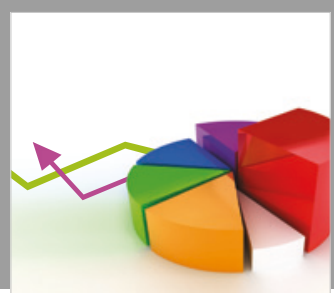

Journal of

Probability and Statistics
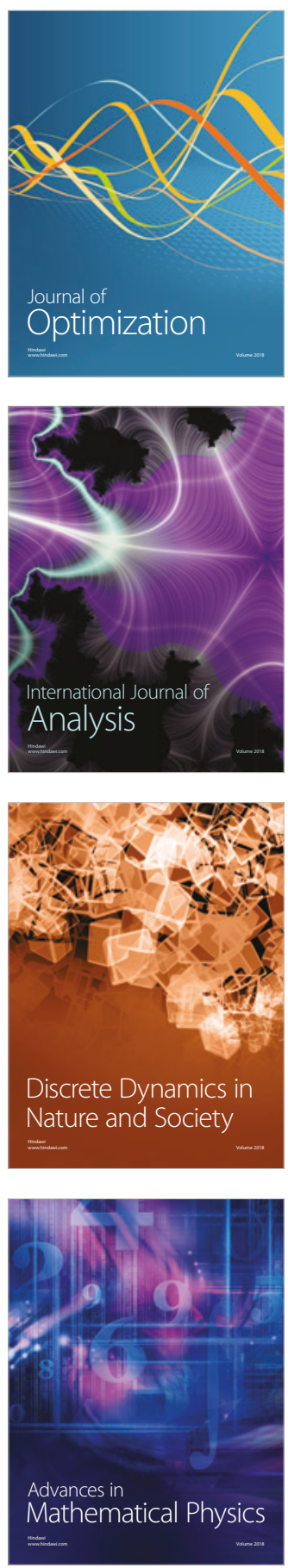